\title{
Heat transport and thermal rectification in molecular junctions: A minimal model approach
}

\author{
E. Díaz, ${ }^{1,2}$ R. Gutierrez, ${ }^{1}$ and G. Cuniberti ${ }^{1,3}$ \\ ${ }^{1}$ Institute for Materials Science, Technische Universität Dresden, D-01062 Dresden, Germany \\ ${ }^{2}$ Departamento de Física de Materiales, GISC, Universidad Complutense, E-28040 Madrid, Spain \\ ${ }^{3}$ Division of IT Convergence Engineering National Center for Nanomaterials Technology, POSTECH, Pohang 790-784, Republic of Korea
}

(Received 8 July 2011; revised manuscript received 1 September 2011; published 4 October 2011)

\begin{abstract}
Heat conduction properties are investigated in a molecular junction modeled as a two-strand ladder with strongly asymmetric thermal transport pathways. By confining anharmonic contributions to only one of the strands, it is shown that tuning of the interstrand coupling can lead to normal heat transport and to the emergence of a well-defined temperature gradient. More interestingly, thermal rectification is obtained around a critical value of the interstrand interaction and by appropriate asymmetries induced by the coupling to the thermal baths.
\end{abstract}

DOI: 10.1103/PhysRevB.84.144302

PACS number(s): 44.10.+i, 05.60.Cd, 66.70.-f

\section{INTRODUCTION}

Energy transfer plays a fundamental role at many different length scales ranging from the macroscopic world down to the molecular level. ${ }^{1,2}$ In particular, the development of nanoscale electronics over the past years and the possibility to design hybrid devices, where biomolecules also could be integrated, 3,4 represent a strong motivation to investigate not only the mechanisms of heat flow in low dimensions as well as in biosystems, but also possible ways to control it. ${ }^{5-9}$ Due to the structural complexity of such systems, the study of heat transport on a first-principle level becomes difficult, and thus, the development of minimal model approaches provides a very useful starting point. ${ }^{10-14}$ In this context, the clarification of issues related to the validity of macroscopic phenomenological laws, e.g., Fourier's law, to describe heat propagation in nanoscale systems has attracted much attention in the field. ${ }^{15,16}$ Thus, for harmonic systems, anomalous heat transportdiverging thermal conductivity with increasing system sizeis obtained, and thus, Fourier's law is not valid. On the contrary, the inclusion of nonlinear local potentials provides a sufficient condition for changing this scenario: ${ }^{17}$ Conservation of the total momentum breaks down, and Fourier's law is revealed as valid. Studies based on the Frenkel-Kontorova (FK) model, ${ }^{18,19}$ the $\phi^{4}$ model, ${ }^{20,21}$ and the Fermi-PastaUlam model ${ }^{22,23}$ have appeared, and potential applications as, e.g., thermal rectifiers or thermal transistors have been proposed, ${ }^{24-26}$ which opens the fascinating possibility for realizing the thermal equivalents to electronic devices.

In this paper, we address heat transport in a molecular junction, see Fig. 1(a), which may be considered as an effective model comprising two vibrational subsystems with strongly different vibrational and, hence, heat conduction properties: One strand includes only harmonic interactions, while the other strand additionally contains local nonlinear potential terms. Although this can be considered as a limiting case, it serves to illustrate the heat transport properties of the system in a clear way; our results are also qualitatively valid in the case where both strands contain nonlinearities as far as the corresponding strengths strongly differ from each other. We expect this effective model to mimic, e.g., the vibrational structure of complex biomolecular systems, where the vibrational properties of specific atomic subsets can be described within the harmonic approximation, while the remaining vibrational degrees of freedom provide a nonlinear environment to which the former system is coupled. We demonstrate that rectification of the heat current is possible by tuning the coupling between the two subsystems and by asymmetries induced through the way the thermal baths are coupled to the system.

\section{MODEL AND METHODOLOGY}

The (dimensionless) Hamiltonian of the minimal model consists of three terms, describing a harmonic $(\mathrm{H})$ strand $\mathcal{H}_{\mathrm{H}}$, an anharmonic $(\mathrm{A})$ strand $\mathcal{H}_{\mathrm{A}}$, and the harmonic coupling between them $\mathcal{H}_{\text {int }}$, respectively. Each strand contains $n=$ $1 \ldots N$ sites, and the intersite interactions, both along each strand as well as between the strands, are assumed to be linear. Nonlinearities are assumed to appear as local potential terms in the A strand within the FK model. The Hamiltonian of the vibrational system is then given by

$$
\begin{aligned}
\mathcal{H} & =\mathcal{H}_{\mathrm{H}}+\mathcal{H}_{\mathrm{A}}+\mathcal{H}_{\text {int }}, \\
\mathcal{H}_{\mathrm{A}} & =\sum_{n=1}^{N} \frac{1}{2} \dot{x}_{n}^{2}+W\left(x_{n}, x_{n-1}\right)+V\left(x_{n}\right), \\
\mathcal{H}_{\mathrm{H}} & =\sum_{n=1}^{N} \frac{1}{2} \dot{y}_{n}^{2}+W\left(y_{n}, y_{n-1}\right), \\
\mathcal{H}_{\text {int }} & =\sum_{n=1}^{N} k_{\text {int }} W\left(x_{n}, y_{n}\right) .
\end{aligned}
$$

Here, the generic term $W\left(x_{n}, x_{m}\right)=\frac{1}{2}\left(x_{n}-x_{m}\right)^{2}$ accounts for harmonic interactions. For the sake of simplicity, we have considered the same elastic constants for both chains, $k$, which define the harmonic frequency $\omega_{0}=k / m$. The interstrand coupling $k_{\text {int }}$ is then expressed in units of $k$. The nonlinear interactions are given by the FK potential $V\left(x_{n}\right)=$ $\left(-V_{0} / 4 \pi^{2}\right) \cos \left(2 \pi x_{n} / b\right)$ with strength $V_{0}$. Furthermore, the period of the FK potential $b$ will be taken as the length scale of the system, and the magnitude $k b^{2}$ will be the energy unit.

Heat transport in this system will be studied by coupling it to thermal baths at different temperatures: cold $\left(T_{C}\right)$ and hot $\left(T_{H}\right)$. The dynamics of the chain coupled to the heat baths is 

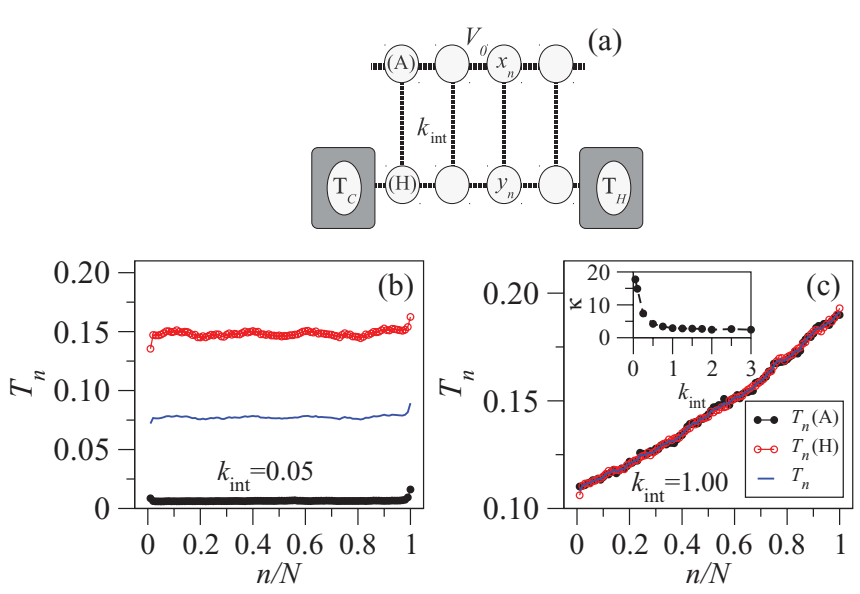

FIG. 1. (Color online) (a) Schematic of the model molecular junction considered in this paper. The vibrational properties of each strand strongly differ: the $\mathrm{H}$ strand includes only $\mathrm{H}$ interactions, while the A strand also contains local nonlinearities. Hence, the thermal conductivity of the whole system will dramatically depend on the interstrand interaction. In the case shown in the figure, the $\mathrm{H}$ chain is connected to thermal baths at temperatures $T_{C}$ and $T_{H}$, respectively. (Bottom panels) Stationary temperature profile for $N=100$ sites connected to two independent heat reservoirs at temperatures $T_{C}=0.1$ and $T_{H}=0.2$. The temperature profile is plotted for a $\mathrm{H} T_{n}(\mathrm{H})$ and an A $T_{n}(\mathrm{~A})$ chain, as well as for the coupled system $T_{n} . V_{0}=5$, (b) $k_{\text {int }}=0.05$ and (c) $k_{\text {int }}=1.00$. The inset shows the thermal conductivity as a function of the coupling $k_{\text {int }}$.

assumed to be described by stochastic Langevin equations, ${ }^{15}$

$$
\begin{aligned}
\frac{d^{2} y_{n}}{d t^{2}}= & -W^{\prime}\left(y_{n}, y_{n-1}\right)-W^{\prime}\left(y_{n}, y_{n+1}\right)-W^{\prime}\left(x_{n}, y_{n}\right) \\
& -\gamma \frac{d y_{n}}{d t}+f_{n}(t),
\end{aligned}
$$

where the last two terms, representing friction and random forces, respectively, are nonzero only for sites $n=1$ and $n=$ $N$ and the prime indicates a derivative with respect to $y_{n}$. The random forces satisfy $\left\langle f_{n}(t) f_{n}\left(t^{\prime}\right)\right\rangle=2 T \gamma \delta\left(t-t^{\prime}\right)$. The dimensionless temperature $T$ is related to the real one $T_{0}$ as $T=k_{B} T_{0} / k b^{2}$, where $k_{B}$ is the Boltzmann constant. The local heat flux, as defined through the continuity equation, can be written as

$$
\begin{aligned}
J_{n}= & \dot{x}_{n}\left(\frac{\partial W^{\prime}\left(x_{n}, x_{n-1}\right)}{\partial x_{n}}+k_{\mathrm{int}} \frac{\partial W^{\prime}\left(x_{n}, y_{n}\right)}{\partial x_{n}}\right) \\
& +\dot{y}_{n}\left(\frac{\partial W^{\prime}\left(y_{n}, y_{n-1}\right)}{\partial y_{n}}+k_{\mathrm{int}} \frac{\partial W^{\prime}\left(x_{n}, y_{n}\right)}{\partial y_{n}}\right) .
\end{aligned}
$$

It is to be noticed that the equations of motion constitute a source of randomness in the problem, and therefore, it is crucial to solve them with a proper numerical method. In our case, we have chosen the procedure proposed by Greenside and Helfand as a correction to the Runge-Kutta method for stochastic equations $\left(3_{O} 4_{S} 2_{G}\right)$, see Refs. 27-29 for further details about the numerical method. Molecular dynamics simulations are performed within open boundary conditions by considering a long enough integration time, around $10^{10}$ time steps such that the stationary state is established. The time step is $\delta t=10^{-5}$, and the friction constant of the baths is set to $\gamma=0.5$ in all simulations. In the final state, the time-averaged heat flux reaches a constant value along the system such that $J=\left\langle J_{1}\right\rangle=\cdots=\left\langle J_{N}\right\rangle$. Thus, the thermal conductivity for a finite system can be calculated as $\kappa=J N /\left(T_{C}-T_{H}\right)$. Similarly, in the steady state, the time-averaged temperature, calculated as $T_{n}=\left\langle\dot{x}_{n}^{2}+\dot{y}_{n}^{2}\right\rangle$, will reach the stationary thermal profile.

\section{HEAT CONDUCTION}

The first bath configuration we will address is depicted in Fig. 1(a). Notice that the A chain can only indirectly influence the heat propagation; its contribution can be tuned via the interstrand interaction $k_{\text {int }}$. Although, for weak interstrand coupling, no thermal gradient will be established in the system, we expect that, by tuning the coupling $k_{\text {int }}$, the A chain will increasingly influence the vibrational dynamics, and thus, a constant thermal gradient would emerge. This transition is shown in the bottom panels of Fig. 1 for a system containing $N=100$ sites, and for two interchain interactions, $k_{\text {int }}=0.05$ and 1.00. The inset of Fig. 1(c) also shows that the thermal conductivity along the system decays as a function of $k_{\text {int }}$, which results from the fact that the anharmonic contributions become stronger with larger coupling leading to an increased thermal resistance. It is worth mentioning that, for large enough coupling $k_{\text {int }}$, in addition to the emergence of a thermal gradient, a length independent thermal conductivity would be expected to occur due to the inclusion of local potential terms in the system. ${ }^{17}$ Accordingly, Fourier's law would be expected to be valid in this parameter regime. Our calculations indeed seem to be consistent with this presumption for systems of size $N \leqslant 200$, far from the thermodynamic limit. However, the simulations needed to satisfactorily show that $\kappa$ is finite for larger systems are beyond our computational resources, and therefore, we cannot present a clear confirmation of this result.

To provide an estimate of the threshold interaction $k_{\text {int }}^{*}$ necessary to create a constant thermal gradient, we introduce two auxiliary systems. First, a reference harmonic ladder with $V_{0}=0$ in Eq. (1) is considered, which has two vibrational bands, an acoustic and an optical one. The latter has dispersion $\bar{\omega}_{\mathrm{H}}^{2}\left(p, k_{\text {int }}\right)=2 k_{\text {int }}+2[1-\cos (p)]$ with $p \in[-\pi, \pi]$ and $\bar{\omega}$ given in units of $\omega_{0}$. An increase in $k_{\text {int }}$, thus, leads to a shift in $\bar{\omega}_{\mathrm{H}}^{2}\left(p, k_{\text {int }}\right)$ to higher frequencies. Notice that, due to the anharmonic interactions, one cannot define vibrational bands in the system of Eq. (1) with $V_{0} \neq 0$ in a rigorous way. However, within the self-consistent phonon theory (SCPT), ${ }^{30}$ the properties of that system can be described in terms of two effective bands. Second, we consider an isolated FK chain, in terms of which the effective optical band of Eq. (1) for $V_{0} \neq 0$ can approximately be described in the limiting case of small $k_{\text {int }}$ (weak band mixing) by using SCPT. This approach consists of replacing the anharmonic potential by an effective harmonic model, whose frequency (or force constant) is temperature dependent, ${ }^{30,31}$

$$
V\left(x_{n}\right)=\frac{-V_{0}}{4 \pi^{2}} \cos \left(2 \pi x_{n}\right) \rightarrow \frac{U(T)}{2} x_{n}^{2} .
$$

By performing a variational study, ${ }^{30}$ the effective interaction $U(T)$ for the Hamiltonian $\mathcal{H}_{\mathrm{A}}$ can be obtained by solving the 

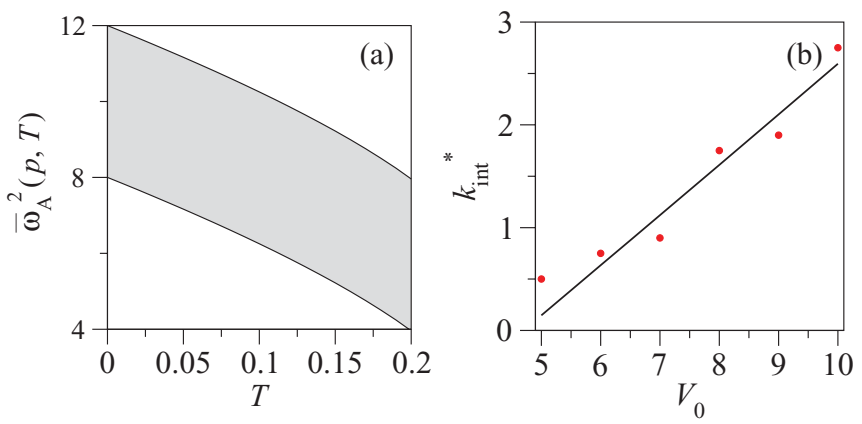

FIG. 2. (Color online) (a) The shadowed region shows the frequency band of states for a FK chain calculated by SCPT as a function of temperature and $V_{0}=8$. (b) Comparison between the analytical estimation of $k_{\mathrm{int}}^{*}$ with the results from numerical simulations for $N=100$ sites and different values of $V_{0}$.

following transcendental equation:

$$
U(T)=V_{0} \exp \left(\frac{-2 T \pi^{2}}{\sqrt{U(T)(U(T)+4)}}\right) .
$$

Once $U(T)$ is known, where $T$ is the average temperature of the A chain, the dispersion relation of the system $\bar{\omega}_{\mathrm{A}}^{2}(p, T)=$ $U(T)+2[1-\cos (p)]$ with $p \in[0, \pi]$ and the density of states (DOS) for the effective harmonic system can be calculated analytically. The frequency band of the SCPT for $V_{0}=8$ as a function of the temperature is presented in the shadowed region of Fig. 2(a). It can be seen that increasing $T$ leads to a softening of $\bar{\omega}_{\mathrm{A}}^{2}(p, T)$.

Going back now to the original system, Eq. (1), it is evident that an increase in $k_{\text {int }}$ will enhance the effect of the bath onto the A chain, which hence, will reach a higher $T$ in the stationary state. As a result, $\bar{\omega}_{\mathrm{A}}^{2}(p, T)$ and $\bar{\omega}_{\mathrm{H}}^{2}\left(p, k_{\mathrm{int}}\right)$ will approach each other. We claim that, when these two bands overlap, then the interchain interaction is large enough to couple the spectra of the $\mathrm{H}$ chain and the A chain, namely, band mixing occurs. Notice that, at $k_{\mathrm{int}}^{*}$, due to the formation of the thermal gradient, the average temperature of the A chain will reach the mean value $T=T_{M}$ between the cold and the hot baths.

Extensive simulations have shown that the overlap condition between the bands $\bar{\omega}_{\mathrm{H}}^{2}\left(p, k_{\text {int }}\right)$ and $\bar{\omega}_{\mathrm{A}}^{2}\left(p, T_{M}\right)$ sensitively depends on the temperature, which is a consequence of the fact that a temperature-induced thermal broadening at high temperatures is beyond the scope of the SCPT. When such effects are negligible, we numerically have confirmed that the thermal gradient arises if approximately half the states are common to the two above-mentioned bands. Thus, the interaction threshold can be estimated by

$$
k_{\mathrm{int}}^{*}=\frac{U\left(T_{M}\right)-2}{2} .
$$

Figure 3 shows the formation of a thermal gradient with increasing $k_{\text {int }}$ for $N=100$. It also presents the analytical DOS [computed within the SCPT using $\bar{\omega}_{\mathrm{A}}^{2}\left(p, T_{A}\right)$ ] of a single A chain at an averaged temperature $T_{A}=\sum_{n} T_{n}(\mathrm{~A}) / N$ as


reference harmonic model with an optical band at $\bar{\omega}_{\mathrm{H}}^{2}\left(p, k_{\text {int }}\right)$ is shown as well (the acoustic band is omitted for simplicity). In the inset of Fig. 3(c), a well-formed thermal gradient is

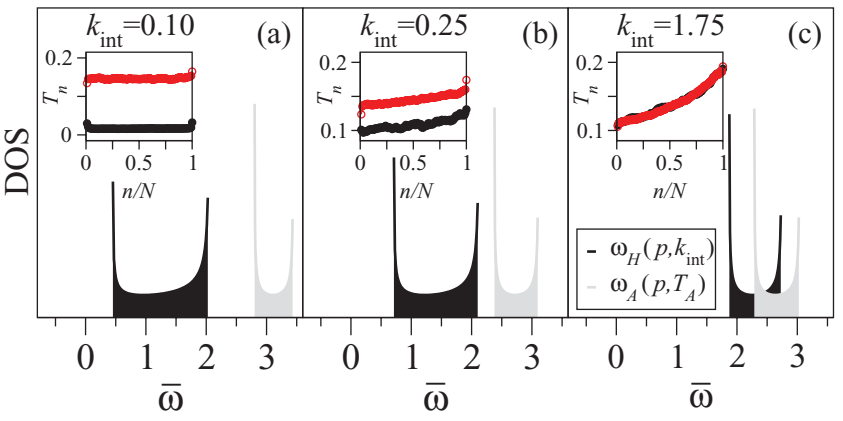

FIG. 3. (Color online) Analytical calculation of the density of states of a single FK chain at temperature $T_{A}$ as well as that of the optical band of a reference harmonic ladder for (a) $k_{\text {int }}=0.1$, (b) $k_{\text {int }}=0.25$, (c) and $k_{\text {int }}=1.75$ and $V_{0}=8$. Insets show the stationary temperature profile for the $\mathrm{H}$ (empty red circles) and the $\mathrm{A}$ chains (filled black circles) for $N=100$ sites connected to two heat reservoirs at $T_{C}=0.1$ and $T_{H}=0.2$.

established when $k_{\mathrm{int}}^{*}=1.75$, for which the DOS of $\bar{\omega}_{\mathrm{H}}^{2}\left(p, k_{\mathrm{int}}^{*}\right)$ and $\bar{\omega}_{\mathrm{A}}^{2}\left(p, T_{A}\right)$ overlap over $50 \%$ of their area. Figure $2(\mathrm{~b})$ demonstrates the good agreement between the analytical estimation of $k_{\mathrm{int}}^{*}$ and that obtained by numerical simulations for different values of $V_{0}$. The latter is obtained by performing several simulations with increasing interstrand coupling up to find a well-formed constant thermal gradient along the system and a length independent thermal conductivity within the numerical resolution.

\section{HEAT RECTIFICATION}

Symmetry breaking and nonlinearities are necessary ingredients to support heat rectification. ${ }^{24-26}$ Here, we demonstrate heat rectification by a topological asymmetry related to the way the thermal baths are coupled to the ladder, similar to what was considered for charge transport in DNA. ${ }^{32}$ Panels (a) and (b) of Fig. 4 show the two considered bath configurations where $T_{H}$ and $T_{C}$ refer to the temperature of the hot and the cold heat reservoirs as previously mentioned. Notice that, in Fig. 4(a), the hot reservoir is connected to the left edge of the A chain, while in Fig. 4(b), the position of the bath connections is reversed. This means that the heat current flows in opposite directions for both cases, but more interestingly, our results show that these currents reach different values in the stationary state, and therefore, the system behaves as a thermal rectifier.

In order to analyze the main features of the heat rectification, we define $\kappa_{+}=J_{+} N /\left(T_{H}-T_{C}\right)$ and $\kappa_{-}=J_{-} N /\left(T_{C}-\right.$ $T_{H}$ ), which are microscopic, possibly chain-length-dependent thermal conductivities. Heat rectification $\kappa_{+}>\kappa_{-}$is clearly demonstrated for a certain range of $k_{\text {int }}$ in Fig. 4(c). The difference between both conductivities $\Delta=\kappa_{+}-\kappa_{-}$reaches a maximum $\Delta_{\max }$ for a coupling $k_{\text {int }}^{0} \sim 0.25$, where a strong rectification effect of about $35 \%$ is found. Qualitatively, this behavior can be understood by the following arguments. When the interstrand coupling is increased from zero, new channels connecting both chains allow for the heat to flow from the hot reservoir to the cold one, and the thermal conductivity increases. More interestingly, our simulations show that $\kappa_{+}$ reaches higher values than $\kappa_{-}$for an interchain coupling 



FIG. 4. (Color online) Schematic of the ladder model connected to two heat reservoirs at temperatures $T_{H}$ and $T_{C}$ in two different configurations (a) and (b). (c) Thermal conductivity $\kappa_{+}$and $\kappa_{-}$for the cases (a) and (b), respectively, as a function of the interchain coupling with $V_{0}=5$ and $N=100$. The inset shows the system-size dependence of $\Delta_{\max }=\kappa_{+}-\kappa_{-}$for $V_{0}=5$. (d) Normalized thermal conductivity difference $\Delta / \Delta_{\max }$ as a function of the coupling $k_{\text {int }}$ for $N=100$ and for $V_{0}=5$ and $V_{0}=8$. Bottom panels present the stationary temperature profile for the coupled system, $N=100$ sites and $V_{0}=5$ connected in the two considered configurations (a) and (b). Two different interchain couplings are considered (e) $k_{\text {int }}=0.1$ and (f) $k_{\text {int }}=2.0$.

smaller than a limiting one $k_{\text {int }}^{\infty}$, where the rectification effects vanish.

For moderate interchain couplings, $k_{\text {int }}<k_{\text {int }}^{\infty}$, because heat transport in a harmonic chain is highly efficient, all sites along that strand quickly reach the temperature of its bath connection. Thus, every harmonic site affects the A chain as an effective local heat reservoir at that temperature. As a result, the stationary thermal profile of the system presents a vanishingly small gradient close to the temperature of the bath connected to the $\mathrm{H}$ chain, except for some deviations close to the opposite bath connection, see Fig. 4(e). Figures 4(e) and 4(f) show the stationary thermal profile along the system for the two considered configurations in Figs. 4(a) and 4(b) and different interchain couplings. In Fig. 4(e), it is shown that, in the case of moderate coupling, there is a clear asymmetry between these two profiles related to the observed heat rectification.

We can get information about the vibrational states involved in the heat transport for moderate $k_{\text {int }}$ by taking these stationary profiles, whose mean value will define the position of the SCPT band of the A strand, into account. As mentioned in Sec. III, the SCPT band of the A chain is shifted to lower frequencies at higher temperatures; hence, when the $\mathrm{H}$ chain is connected to the hot bath [Fig. 4(b)], we find an increase in the spectral

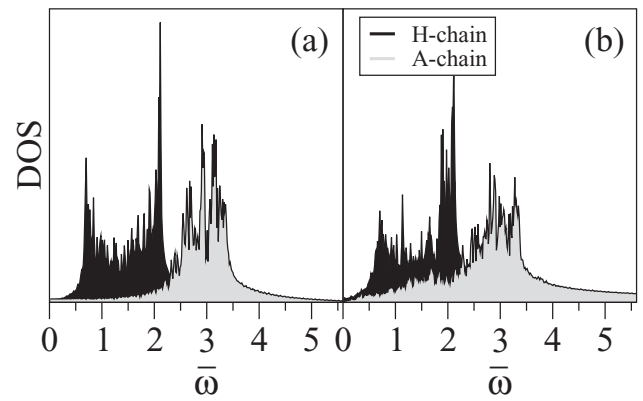

FIG. 5. Numerical calculation of the density of states of the $\mathrm{H}$ (black) and the A (gray) strands in the configurations (a) and (b) of Fig. 4 for $k_{\text {int }}=0.5$ and $V_{0}=8$. Notice the increased DOS of the A strand at the low-frequency region in the case of Fig. 4(b).

density of anharmonic modes in the low-frequency domain, see Fig. 5(b). Since the main carriers of the heat are low-frequency modes, this increased spectral density will lead to an enhanced energy exchange with the A strand and to a global increase in the thermal resistance.

On the contrary, when the connections to the thermal baths are reversed [Fig. 4(a)], the A chain reaches a colder temperature, and its SCPT band shifts to higher frequencies and so does the low-energy part of its spectral density. Thus, low-frequency anharmonic states will hardly be activated by the temperature, and the transport process is mainly supported by the $\mathrm{H}$ strand, see Fig. 5(a). As a result of this behavior, heat transport mainly occurs through the $H$ chain in the configuration of Fig. 4(a), while the thermal conductivity includes much stronger contributions from the anharmonic interactions in case of Fig. 4(b). Given the fact that the thermal resistance increases with the anharmonicity, it is expected that $\kappa_{+}>\kappa_{-}$, which is consistent with our numerical results. It should be noticed that, by considering larger systems, the rectification rate increases, see the inset of Fig. 4(c). This is because the local bath effects created by the $\mathrm{H}$ chain, and which are responsible for the asymmetry of the stationary thermal profiles in Fig. 4(e), become stronger.

If $k_{\text {int }}$ is large enough, the increasing dominance of the nonlinear interactions and band mixing will unify the properties of systems (a) and (b), and it will lead to a decrease in the thermal conductivity, see the inset of Fig. 1(c). Therefore, the final thermal gradient of both situations will basically be the same as shown in Fig. 4(f), and asymptotically, the heat rectification will be suppressed. In Fig. 4(c), we show that this situation occurs for $k_{\text {int }}>k_{\text {int }}^{\infty} \sim 1.0$.

How is the strength $V_{0}$ of the anharmonic interactions influencing the rectification behavior? As shown in Fig. 4(d), where $\Delta / \Delta_{\max }$ is plotted as a function of $k_{\text {int }}$ for two different anharmonicities, the qualitative behavior does not depend on $V_{0}$, but the main rectifying features are shifted to larger $k_{\text {int }}$. This is consistent with the fact that band mixing in Fig. 1(a) occurs for larger couplings when the anharmonicity present in the system, is strengthened. As a result, we demonstrated that $k_{\text {int }}^{*}$ increases with the nonlinearity $V_{0}$. Although it is not possible to establish a direct relationship between $k_{\text {int }}^{\infty}$ and $k_{\text {int }}^{*}$, the results of the simulations hint at the relation $k_{\text {int }}^{\infty}>k_{\text {int }}^{*}$. Therefore, we can predict that the main rectification effects are expected to appear for $k_{\text {int }}<k_{\text {int }}^{*}$. 


\section{CONCLUSIONS}

We have investigated how normal heat conduction can emerge in a molecular junction modeled via a two-strand ladder with strongly different vibrational spectra on each strand. Hereby, the interstrand coupling has turned out to be the key parameter. Analytical and numerical estimations have been provided for the threshold interaction at which a transition to normal heat transport occurs. We have estimated this threshold by considering the self-consistent phonon theory for a single A chain in comparison to a fully harmonic ladder model. The analytical results were in good agreement with numerical results based on nonequilibrium molecular dynamics simulations.

Taking advantage of the nonlinear effects present in the system, it was shown that heat current behaves differently if the thermal bath connections are interchanged between the harmonic and the anharmonic subsystems. This mechanism provides strong heat rectification of more than $30 \%$. The main qualitative features of the rectifying device do not depend on the system size nor on the strength of the anharmonicity. However, the maximum rectification rates increase with the system size and shift to larger couplings in the case of stronger anharmonic interactions. Our results remain valid for temperatures high enough to activate the high-frequency optical band derived from the anharmonic interactions within the regime of validity of the SCPT.

Our minimal model is expected to be relevant in the study of thermal conduction in complex biomolecular systems where two vibrational subsystems with different degrees of anharmonicity can interact with each other. In particular, within the discussed two-strand ladder, thermal properties of double- or multiple-stranded molecules, such as DNA molecules or $\alpha$ helices in proteins, can be described. ${ }^{11-13}$

\section{ACKNOWLEDGMENTS}

This work was supported by DFG-Projekt CU 44/20-1 Project No. MAT2010-17180 and by the South Korea Ministry of Education, Science and Technology Program "World Class University" under Contract No. R31-2008-000-10100-0. E.D. acknowledges financial support by MEC and the program Flores Valles-UCM. We acknowledge fruitful discussions with H. Sevincli and S. Avdoshenko.
${ }^{1}$ D. M. Leitner, Annu. Rev. Phys. Chem. 59, 233 (2008).

${ }^{2}$ F. Giazotto et al., Rev. Mod. Phys. 78, 217 (2006).

${ }^{3}$ N. C. Seeman, Mol. Biotechnol. 37, 246 (2007).

${ }^{4}$ I. Medalsy et al., Nano Lett. 8, 473 (2008).

${ }^{5} \mathrm{Z}$. Wang et al., Science 317, 787 (2007).

${ }^{6}$ D. Y. Li et al., Appl. Phys. Lett. 83, 2934 (2003).

${ }^{7}$ W. Li, H. Sevincli, G. Cuniberti, and S. Roche, Phys. Rev. B 82, 041410(R) (2010).

${ }^{8}$ L.-A. Wu and D. Segal, Phys. Rev. Lett. 102, 095503 (2009).

${ }^{9}$ D. Segal and A. Nitzan, Phys. Rev. Lett. 94, 034301 (2005).

${ }^{10}$ K. Iguchi, Int. J. Mod. Phys. B 11, 2405 (1997).

${ }^{11}$ E. Díaz, A. Sedrakyan, D. Sedrakyan, and F. Domínguez-Adame, Phys. Rev. B 75, 014201 (2007).

${ }^{12}$ A. V. Savin, M. A. Mazo, I. P. Kikot, L. I. Manevitch, and A. V. Onufriev, Phys. Rev. B 83, 245406 (2011).

${ }^{13}$ D. Hennig, Phys. Rev. B 65, 174302 (2002).

${ }^{14}$ W.-R. Zhong, Phys. Rev. E 81, 061131 (2010).

${ }^{15}$ S. Lepri, R. Livi, and A. Politi, Phys. Rep. 377, 1 (2003).

${ }^{16}$ A. Dhar, Adv. Phys. 57, 457 (2008).

${ }^{17}$ B. Hu, B. Li, and H. Zhao, Phys. Rev. E 57, 2992 (1998).

${ }^{18}$ G. P. Tsironis, A. R. Bishop, A. V. Savin, and A. V. Zolotaryuk, Phys. Rev. E 60, 6610 (1999).
${ }^{19}$ W.-R. Zhong, P. Yang, B.-Q. Ai, Z.-G. Shao, and B. Hu, Phys. Rev. E 79, 050103(R) (2009).

${ }^{20}$ B. Hu, B. Li, and H. Zhao, Phys. Rev. E 61, 3828 (2000).

${ }^{21}$ F. Piazza and S. Lepri, Phys. Rev. B 79, 094306 (2009).

${ }^{22}$ K. Saito and A. Dhar, Phys. Rev. Lett. 104, 040601 (2010).

${ }^{23}$ Z. Liu, X. Wu, H. Yang, N. Gupte, and B. Li, New J. Phys. 12, 023016 (2010).

${ }^{24}$ M. Terraneo, M. Peyrard, and G. Casati, Phys. Rev. Lett. 88, 094302 (2002).

${ }^{25}$ B. Hu, L. Yang, and Y. Zhang, Phys. Rev. Lett. 97, 124302 (2006).

${ }^{26}$ L. Zhang, J.-S. Wang, and B. Li, Phys. Rev. B 81, 100301(R) (2010).

${ }^{27}$ E. Helfand, Bell Syst. Tech. J. 58, 2289 (1979).

${ }^{28}$ H. S. Greenside and E. Helfand, Bell Syst. Tech. J. 60, 1927 (1981).

${ }^{29}$ J. Casado-Pascual, C. Denk, J. Gómez-Ordóñez, M. Morillo, and P. Hänggi, Phys. Rev. E 67, 036109 (2003).

${ }^{30}$ T. Dauxois, M. Peyrard, and A. R. Bishop, Phys. Rev. E 47, 684 (1993).

${ }^{31}$ Z.-G. Shao, L. Yang, W.-R. Zhong, D.-H. He, and B. Hu, Phys. Rev. E 78, 061130 (2008).

${ }^{32}$ R. Gutierrez, S. Mohapatra, H. Cohen, D. Porath, and G. Cuniberti, Phys. Rev. B 74, 235105 (2006). 